\title{
Establishment of rat brain endothelial cells susceptible to rat cytomegalovirus ALL-03 infection
}

\begin{abstract}
Endothelial cells have been implicated as key cells in promoting the pathogenesis and spread of cytomegalovirus (CMV) infection. This study describes the isolation and culture of rat brain endothelial cells (RBEC) and further evaluates the infectious potential of a Malaysian rat CMV (RCMV ALL-03) in these cultured cells. Brain tissues were mechanically fragmented, exposed to enzymatic digestion, purified by gradient density centrifugation, and cultured in vitro. Morphological characteristics and expression of von Willebrand factor (factor VIII-related antigen) verified the cells were of endothelial origin. RBEC were found to be permissive to the virus by cytopathic effects with detectable plaques formed within $7 \mathrm{~d}$ of infection. This was confirmed by electron microscopy examination which proved the existence of the viral particles in the infected cells. The susceptibility of the virus to these target cells under the experimental conditions described in this report provides a platform for developing a cell-culture-based experimental model for studies of RCMV pathogenesis and allows stimulation of further studies on host cell responses imposed by congenital viral infections.
\end{abstract}

Keyword: Cytopathology; Isolation; Endothelial cells; Rat cytomegalovirus 